\title{
Towards dual recycling with the aid of time and frequency domain simulations
}

\author{
M Malec ${ }^{1}$, H Grote ${ }^{1}$, A Freise $e^{2}$, G Heinzel ${ }^{1}$, K A Strain ${ }^{3}$, J Hough $^{3}$ \\ and K Danzmann ${ }^{1}$ \\ ${ }^{1}$ Max-Planck-Institute for Gravitational Physics (Albert-Einstein-Institute) and University of \\ Hannover, Callinstr. 38, D-30167 Hannover, Germany \\ ${ }^{2}$ European Gravitational Observatory, Via E Amaldi, 56021 Cascina (PI), Italy \\ ${ }^{3}$ Department of Physics and Astronomy, University of Glasgow, Glasgow G12 8QQ, UK \\ E-mail: icm@aei.mpg.de
}

Received 1 September 2003

Published 11 February 2004

Online at stacks.iop.org/CQG/21/S991 (DOI: 10.1088/0264-9381/21/5/091)

\begin{abstract}
Dual recycling, the combination of the interferometric techniques of power and signal recycling, allows the improvement of the shot noise limited sensitivity of interferometric gravitational wave detectors. GEO 600 is the first km-scale gravitational wave detector using dual recycling. The hardware installation is completed and dual recycling has become a great challenge in terms of commissioning of GEO 600. Simulations show that lock acquisition of the optical system can only be achieved in certain detector states. Thus as we need to start with a locked detector in such a specific state, an appropriate strategy is needed to change the state of detector operation without losing lock. The basic concepts and first results based on time and frequency domain simulations will be presented in this paper.
\end{abstract}

PACS numbers: 04.80.-y, 07.60.Ly, 07.60.Nn, 42.25.Bs, 42.25.Hz, 95.55.Ym, 95.75.Kk, 95.85.Sz

(Some figures in this article are in colour only in the electronic version)

\section{Introduction}

All current laser-interferometric gravitational wave detectors are Michelson interferometers measuring small differential phase modulations of the light in the two arms. One of the limits to the sensitivity is the shot noise associated with the photon statistics. To reduce this effect, all interferometric detectors use the technique of power recycling, which enhances the laser power inside the interferometer arms. One option to become even more sensitive is to influence the storage time of the phase modulation signal sidebands induced by a gravitational wave. For this purpose, Meers [Meers88] suggested the technique of signal recycling in combination with power recycling, so-called dual recycling. The British-German project 


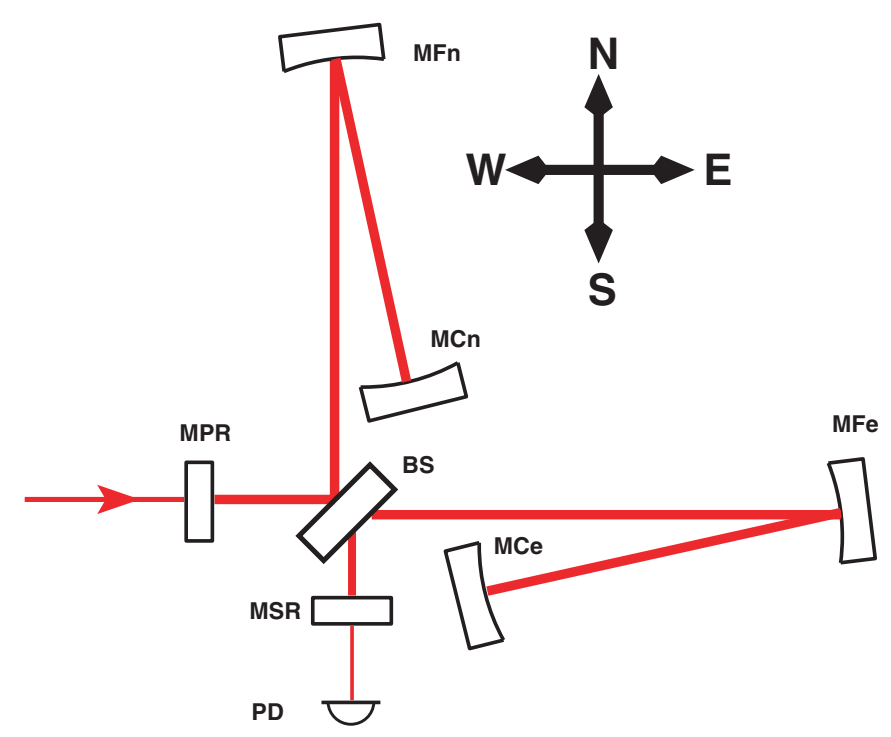

Figure 1. Simplified dual-recycled set-up of GEO 600. The modecleaners and the laser were omitted for clarity.

GEO 600 is the first large-scale gravitational wave detector to incorporate this technique [Willke02]. Figure 1 shows the main set-up of GEO 600. The folded, $600 \mathrm{~m}$ long arms of the Michelson interferometer are adjusted differentially to reflect all laser light back towards the light source at the west port yielding a dark fringe at the south port. Together with the power recycling mirror MPR, the Michelson interferometer forms the so-called power recycling cavity, enhancing the laser light inside the interferometer arms.

The south port will, however, not appear dark for noise or gravitational wave sidebands in the audio range. Thus, by closing the output port of the Michelson interferometer with the MSR mirror, the signal amplitude can be accumulated resonantly inside the signal recycling cavity. Different user specified frequencies of maximum signal enhancement can be selected by tuning the signal recycling cavity length via the position of MSR. The detector state with a maximum sensitivity at DC is called tuned, else detuned.

For the operation of the detector three lengths need to be controlled: the differential armlength of the Michelson interferometer and the lengths of the power recycling and signal recycling cavities. The error signals are generated by various techniques based on phase modulation of the laser light. More detail on the control loops can be found in [Grote3a].

The other large-scale gravitational wave detectors (LIGO in the US, the French-Italian VIRGO project and TAMA 300 in Japan) use the power recycling technique in combination with cavities in the arms of their Michelson interferometers leading to four lengths that need to be controlled. In LIGO, after A/D conversion of all sensor signals, a sensor transformation matrix is digitally calculated which in turn generates feedback signals [Evans01, Heefner01].

To date, trading off the bandwidth and frequency of maximum sensitivity at GEO 600, we aim for a final detuning in the order of $\sim 100 \mathrm{~Hz}$. However, simulations show that locking the detector directly at that operating point is very difficult. It is much easier in a more largely detuned state [Grote03a, b]. Therefore a suitable strategy could be to first catch and lock the mirrors of a detector detuned by for example $20 \mathrm{kHz}$, and then continuously shift the detuning down to the desired value. This technique was first investigated at the Garching 30 m prototype [Heinzel99]. 
To implement this transition, changes of error signal characteristics for each signal recycling state need to be tracked within a huge parameter space. Simulations in the frequency domain give important information about correlations inside the detector under quasi-static conditions. However, the dynamic process of lock acquisition can only be predicted with time domain simulations. Basic concepts and first numerical results of simulations in both domains will now be presented.

\section{Frequency domain simulations}

Once the detector is locked with a detuning of, say, $20 \mathrm{kHz}$, it can be regarded as being in a quasi-static state. Predictions for a slow continuous movement of MSR without the loss of lock can therefore be made with a frequency domain simulation program. For this kind of analysis, FINESSE, a frequency domain interferometer simulation program, was developed within GEO 600 [Finesse, Freise03].

In order to tune MSR in a controlled way, the correlation between the modulation sideband frequency and the position of MSR needs to be investigated. Moreover, the dependence of all error signals on the MSR tuning has to be examined.

\subsection{Systematic movement of the signal recycling mirror}

If all of the interferometer control systems are operating, apart from that for the signal recycling mirror, the remaining uncontrolled degree of freedom is essentially equivalent to the position of the signal recycling mirror. The operating point is then defined by a zero crossing of the signal recycling error signal with respect to mirror position. The ability of the servosystem to hold the mirror at the operating point is determined mainly by the slope of the signal near the operating point, for a given feedback loop gain, and the range of mirror displacement for which there is a restoring force on the mirror. The modulation frequency determines the position of the zero crossings of the error signal and therefore the possible locations to which the mirror can be locked [Freise00]. Thus, slowly detuning the modulation frequency by an amount $\delta f$ will gradually move MSR by some distance $\delta x$.

Figure 2 shows the magnitude of the error signal for the signal recycling feedback loop as a function of MSR position and modulation frequency: every horizontal cut corresponds to the more common, two-dimensional error signal magnitude with respect to the MSR position for a fixed modulation frequency. The displacement $\delta x$ of MSR, measured from the 'tuned' point, is expressed as an angle $\delta \chi=360^{\circ} \cdot \delta x / \lambda$, with $1064 \mathrm{~nm}$ being the laser wavelength $\lambda .\left( \pm 180^{\circ}\right.$ corresponds to a sensitivity maximum at $125 \mathrm{kHz}$.) The modulation frequency axis is expressed as detuning $\delta f$ from the modulation frequency which would give a locking point at the tuned situation. The demodulation phase is adjusted to give a symmetric error signal shape of highest possible horizontal slope at the origin of figure 2; this demodulation phase is kept constant throughout the complete figure. The error signal has been mapped nonlinearly to the greyscale density to give the clearest qualitative representation of the data: at the bright regions the error signal is positive, and at the dark regions it is negative. Therefore the transitions between bright and dark regions mark the zero crossings. Shifting $\delta f$ adiabatically from $-20 \mathrm{kHz}$ to $0 \mathrm{~Hz}$ while locking on one of the diagonal lines of zero crossings allows for a slow continuous sweep of the MSR position from a large detuning $\left(\delta \chi= \pm 30^{\circ} \hat{=} \pm 20 \mathrm{kHz}\right)$ to the tuned state. The diagonals indicate a linear relation between $\delta f$ and $\delta \chi$ with $\delta \chi= \pm 180^{\circ} / F S R_{\mathrm{SR}} \cdot \delta f$, with $F S R_{\mathrm{SR}} \approx 125 \mathrm{kHz}$ being the free spectral range of the signal recycling cavity.

For each fixed modulation frequency, the only variable left to optimize the error signal is the demodulation phase. This phase influences all attributes of the demodulated signal: the 


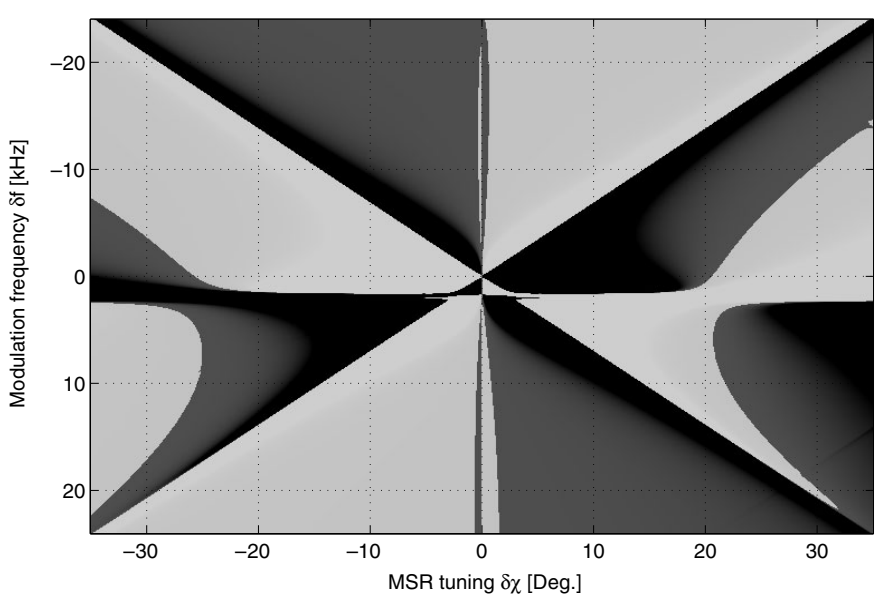

Figure 2. Error signal magnitude for the signal recycling feedback loop in the plane of the MSR position and the modulation frequency, given in detuning $\delta f$ from the specific frequency for a tuned detector. (The demodulation phase is kept constant for all $\delta f$.) The displacement $\delta \chi$ of MSR is given in degrees from the tuned case, with $180^{\circ}$ corresponding to a detuning of half a wavelength $(\lambda / 2=532 \mathrm{~nm})$ or $125 \mathrm{kHz}$ in terms of detector sensitivity. The locations of the zero crossings of the error signal are at the crossovers between bright and dark regions. Continuously transferring the detector from a detuned to tuned state without losing lock means to trace one of the diagonal lines towards the origin by adiabatically sweeping the modulation frequency.

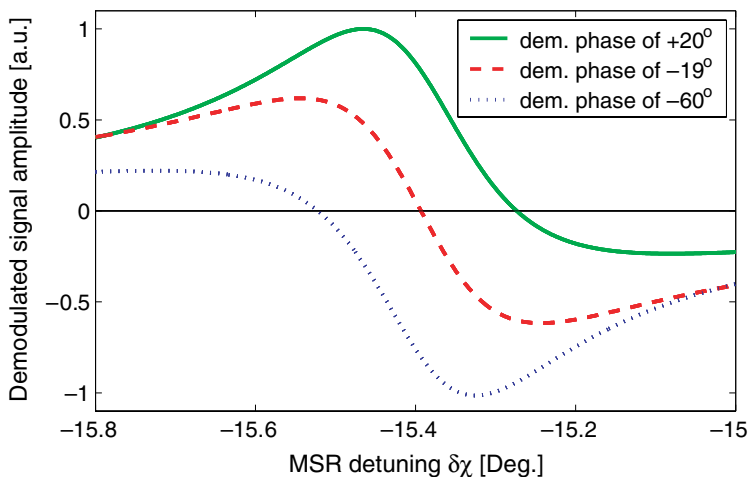

Figure 3. Characteristics of the error signal magnitude for a detector detuned by roughly $11 \mathrm{kHz}$. The only parameter that differs is the demodulation phase changing the main characteristics as for example the position of the zero crossing and its slope.

exact position of the zero crossing and the capture range of the subsequent feedback loop, but particularly the slope at the zero crossing.

Figure 3 shows the characteristics of some signal recycling error signals for the detuned detector with various demodulation phases. With feedback operating, the error signal amplitude corresponds to the force acting on the mirror at the specific mirror position. Even if locked, a certain symmetric rms movement of MSR around the detuned operating point will still remain. If this is just slightly smaller than the capture range of the error signal, the mirror could easily exceed the capture range when the shift of the error signal pattern along the MSR position axis was too fast. This will finally limit the maximum allowed velocity of the signal 

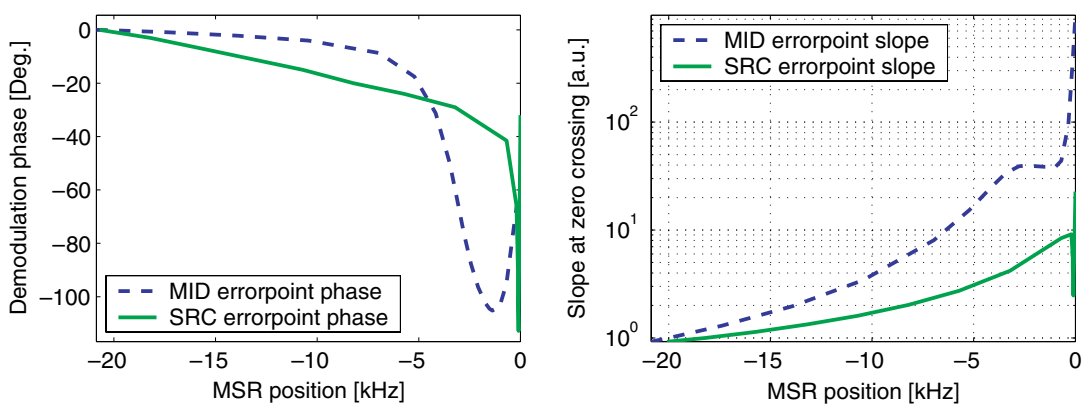

Figure 4. Optimized demodulation phases and the corresponding slopes at the zero crossings of the error signals of the Michelson interferometer (MID) and the signal recycling cavity (SRC). The signal recycling mirror MSR was detuned according to the diagonal line of the upper left quadrant of figure 2 .

recycling cavity tuning. Therefore, the criterion for optimization of the demodulation phase should be to maximize the overall signal and hence the restoring force on each side of the zero crossing. This corresponds to the integral of the error signal: the respective smaller area under the error signal graph left or right from the zero crossing should be maximized. In figure 3 this is achieved with a demodulation phase of $-19^{\circ}$ making the graph symmetric around the zero crossing.

\subsection{Demodulation phase and error signal slope changes during the tuning of MSR}

The determination of the optimum demodulation phase for the signal recycling loop, as suggested in section 2.1, requires some numerical calculation. To simplify the approach, the symmetry of the error signal extreme values to both sides of the zero crossing was chosen as a criterion. The case of the demodulation phase of $-19^{\circ}$ in figure 3 suggests this to be a good choice: the symmetry of the signal evidently satisfies the requirements stated in section 2.1. However, simulations show that this coincidental symmetry disappears for absolute detunings of the operation point smaller than $\approx 7 \mathrm{kHz}$ : as figure 2 shows, there is a permanent zero crossing at $\delta \chi=0^{\circ}$ for every modulation frequency. If near the tuned state, this zero crossing forces the error signal to reach zero more rapidly at the zero crossing's side. This narrows the error signal pattern unidirectionally. Therefore within the tuning interval of $[-7 \mathrm{kHz}, 7 \mathrm{kHz}]$, further simulation fulfilling the necessary conditions mentioned in section 2.1 might lead to a better choice of demodulation phases.

For the Michelson interferometer loop the corresponding mirrors can be assumed to be at rest. Therefore the optimization criterion for the demodulation phase is rather a maximum confinement or slope at the zero crossing. The modulation frequency is kept constant.

Ideally, the carrier light is completely reflected towards MPR by the Michelson interferometer (see section 1) and thus unaffected by any MSR movement. For all modulation sidebands, however, the dual recycled detector forms an optical system of two cavities coupled at the beamsplitter via the armlength difference of the Michelson interferometer. The phase of the sideband light fields therefore depends on both the sideband's frequency and the state of the complete detector. Hence a change of the MSR position will in general result in shifts of the ideal demodulation phase and a change of the error signal gain.

Figure 4 shows the optimized demodulation phases and the corresponding slopes of the Michelson and signal recycling error signals. For this, the signal recycling mirror MSR was 

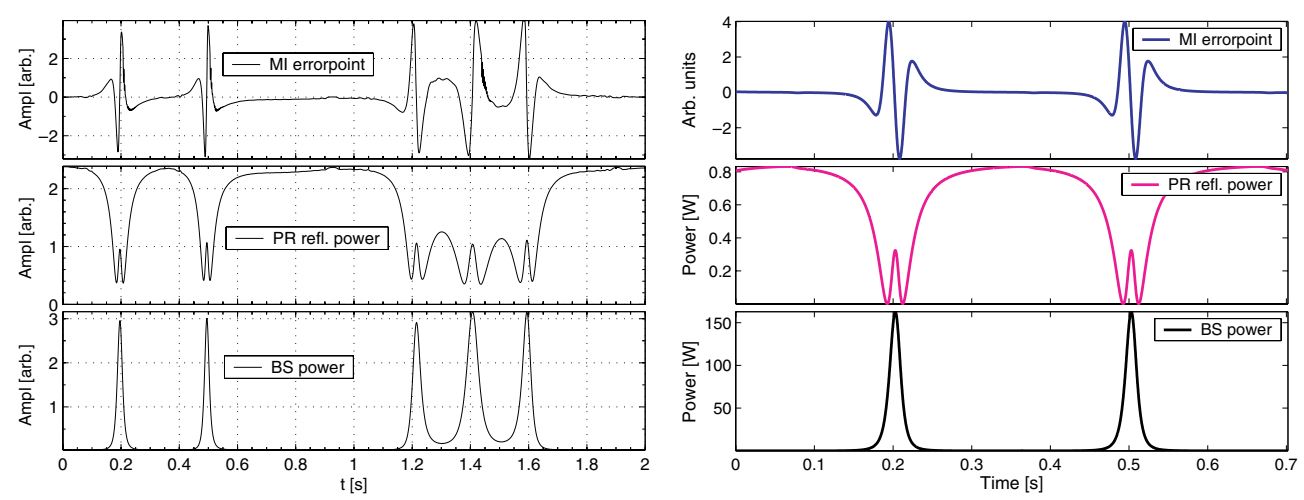

Figure 5. Measurement with the power recycled GEO 600 detector from autumn 2002 (left) and a corresponding simulation with E2E (right). The power recycling cavity is locked whereas the end mirrors MCe and MCn move freely.

detuned according to the diagonal line of the upper left quadrant of figure 2. While the power recycling cavity is hardly affected by the MSR position (and thus omitted in figure 4), at least the Michelson interferometer control will very likely need an automatic gain and phase control: under the terms of the preliminary results, this might also be true for the signal recycling cavity. A micro-controller could tune all parameters simultaneously according to the simulation results. The maximum speed of the tuning will, however, need heuristic investigation.

\section{Time domain simulations}

In order to predict the dynamic behaviour of GEO 600 during lock acquisition, the time domain simulation 'end to end' model (E2E) developed by LIGO [E2E] is utilized. E2E is able to take into account different aspects of an experiment such as optical, mechanical and electronical features. This allows the reconstruction and test of dynamic features of an experiment including feedback loops.

The GEO 600 model built with E2E is kept as simple as possible. So far it contains the complete power recycled Michelson interferometer with a $\mathrm{TEM}_{00}$ field neglecting any curvature mismatch.

One of the main simplifications in comparison with the real set-up are the suspension characteristics which are applied only at the end mirrors. Furthermore, the suspensions are reduced to the most basic needs for a sensible feedback: a digital filter with a complex pole pair represents the last pendulum stage with the mirror as the lower mass. In reality the end mirrors are suspended as triple pendulums with various mode couplings into longitudinal motion [Gossler03].

A fundamental difference to the real experiment is the power recycling cavity feedback loop acting on MPR instead of the laser frequency [Grote3a]. This was necessary due to the time domain property of E2E: changes of the default laser frequency cause difficulties, because every time domain simulation acts as a numeric integrator. In order to accurately calculate any function changing with frequency $\Delta f$, a time step smaller than $0.1 / \Delta f$ is required [Bhawal98]. This, however, may result in long simulation times.

Figure 5 shows (left) a measurement made with the power recycled GEO 600 detector and (right) a corresponding simulation with the E2E model. In both cases the power recycling 


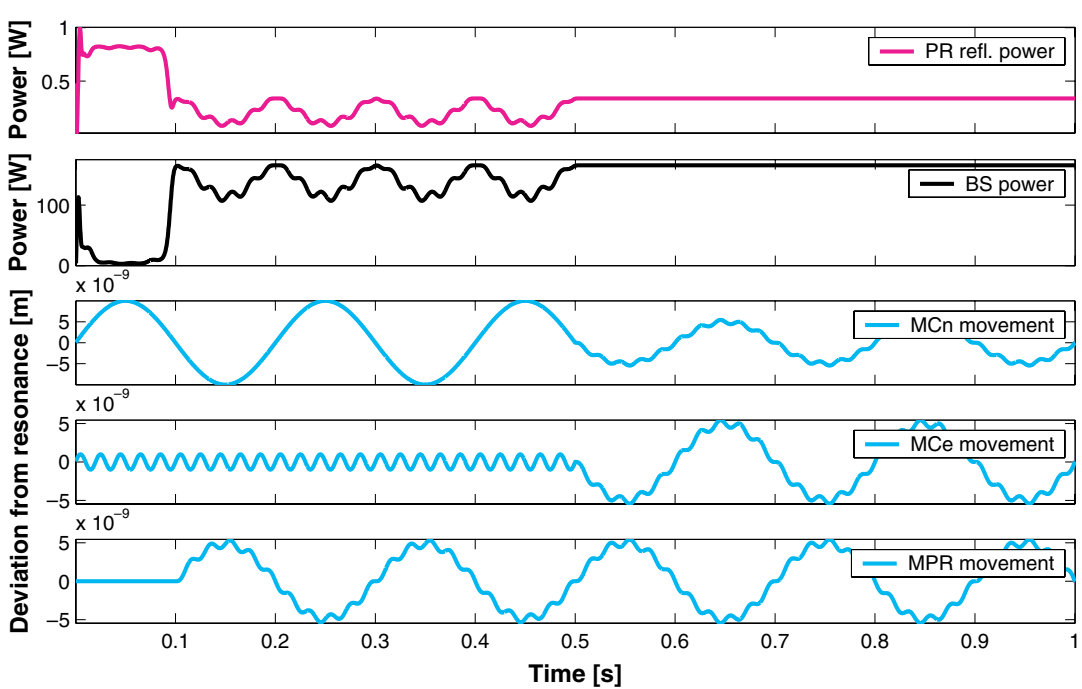

Figure 6. An example of a successful power recycling and Michelson lock acquisition simulated with E2E. MCe and MCn are excited sinusoidally, while MPR is at rest. After $0.1 \mathrm{~s}$ the power recycling cavity feedback is switched on automatically. This moves MPR to keep the power recycling cavity on resonance. The wiggles visible in the intracavity and reflected power are caused by the different coupling states of the power recycling cavity due to the end mirror movements. They vanish at time $0.5 \mathrm{~s}$, when the Michelson differential feedback is turned on. Since the sinusoidal excitations of MCe and MCn still take place, the motion is now spread to all mirrors.

cavity is locked whereas the end mirrors MCe and MCn in principle move freely. This means that the changes in length of the power recycling cavity induced by MCe and MCn motions are compensated, but the reflectivity of the Michelson interferometer still changes with time. From bottom up both parts of figure 5 show the power build-up at the beamsplitter, the power detected in reflection of MPR and the error signal for the Michelson interferometer. The shape of the reflected power occurs due to the transition of the power recycling cavity between undercoupled, impedance matched and overcoupled resonator. Relative changes of the Michelson interferometer armlengths on small time scales can be determined by the width of the power enhancement peak inside the cavity or, at larger time scales, the time gap between the fringes. In this case, both yield a velocity of $1.8 \mu \mathrm{m} \mathrm{s}^{-1}$, which was applied to MCn within the simulation.

Figure 6 shows an example of a successful lock acquisition of power recycling and Michelson simulated with E2E. Initially MCe and MCn are swinging sinusoidally, while MPR is at rest. After $0.1 \mathrm{~s}$ the power recycling cavity feedback is switched on automatically (by performance of a power threshold criterion of the reflected power). This moves MPR to keep the power recycling cavity on resonance. The wiggles visible in the intracavity and reflected power are caused by the different coupling states of the power recycling cavity due to the end mirror movements. They vanish while enabling the Michelson differential feedback. Since the sinusoidal excitations of MCe and MCn still take place, they are now spread among the motion of all mirrors.

The implementation of signal recycling lock is the next thing to follow. E2E simulations might then help to optimize the lock acquisition of dual recycling. 


\section{Acknowledgments}

The authors acknowledge the support of PPARC in the UK, the BMBF, the DFG and the European Graduate College. They would also like to thank LIGO for providing the End to End model simulation program.

\section{References}

[Bhawal98] Bhawal B et al 1998 http://www.ligo.caltech.edu/ e2e/pdfs/T980051_GettingStarted.fm5.pdf15 [E2E] Yamamoto $\mathrm{H}$ et al http://www.ligo.caltech.edu/ $\mathrm{e} 2 \mathrm{e} /$

[Evans01] Evans M et al 2001 Opt. Lett. 27 598-600

[Finesse] Freise A http://www.rzg.mpg.de/ $\sim$ adf/

[Freise00] Freise A et al 2000 Phys. Lett. A 277 135-42

[Freise03] Freise A 2004 Class. Quantum Grav. 21 S1067

[Gossler03] Gossler S 2004 Class. Quantum Grav. 21 S923

[Grote03a] Grote H 2004 Dual recycling for GEO 600 Class. Quantum Grav. 21 S473

[Grote03b] Grote H 2003 http://www.amps.uni-hannover.de/dissertationen/grote_diss.pdf

[Heefner01] Heefner J et al 2001 http://arxiv.org/abs/physics/0111027

[Heinze199] Heinzel G 1999 PhD Thesis MPQ-243

[Meers88] Meers B 1988 Phys. Rev. D 38 2317-26

Meers B 1988 Phys. Rev. D 38 1391-4

[Willke02] Willke B et al 2002 Class. Quantum Grav. 19 1377-87 